\title{
Rheumatoid arthritis in a mouse?
}

\author{
Gary S Firestein
}

Rheumatoid arthritis (RA) remains a uniquely human disease. Dozens of preclinical arthritis models have been developed in a variety of species (e.g. mouse, rat, rabbit and monkey) that involve spontaneous or induced synovial inflammation. None of these, however, is truly $\mathrm{RA}$, and none consistently predicts the effect of a therapeutic agent in patients.

Researchers in academia and the pharmaceutical industry are often led astray by relying too much on animal models. For instance, interleukin-6 deficiency has little or no effect in passive transfer models of arthritis or in tumor necrosis factor transgenic mice, and methotrexate is only marginally effective in collageninduced arthritis (CIA). Anti-CD20 antibodies only work when administered very early in CIA, but not in established disease. For all these drugs, considering the model results without clinical context could have lead investigators to abandon an effective therapeutic approach. Conversely, positive data in rodents might lead to overestimation of the therapeutic effect in humans; for example, NSAIDs are remarkably effective in rat adjuvant arthritis, but provide only modest relief in RA. A comprehensive review has provided an outstanding summary of how various therapeutic interventions affect the disease course in standard animal models (Hegen Met al. [2008] Ann Rheum Dis 67: 15051515), and another discusses cytokine profiles in animal models in relation to RA (Firestein GS et al. [2004] J Clin Invest 114: 471-474).

Animal models have a major place in our preclinical armamentarium, and drug development would be impossible without them. Preclinical models of RA can be used to test very specific hypotheses about the mechanisms of therapeutic agents. For instance, a drug that modulates innate immunity could be evaluated in animal models that are dependent on this arm

\section{Dozens of preclinical arthritis models have been developed... . none of these, however, is truly RA, and none consistently predicts the effect of a therapeutic agent in patients.}

GS Firestein is

Professor of Medicine, Chief of the Division of Rheumatology, Allergy and Immunology, and Dean of Translational Medicine at the University of California, San Diego School of Medicine, CA, USA.

\section{Competing interests \\ The author declared no} competing interests.

www.nature.com/clinicalpractice doi:10.1038/ncprheum0973 of the immune system, such as streptococcal cell wall arthritis or passive K/BxN serum arthritis. If the compound is not effective in these models, then the approach should probably be questioned. A therapy that inhibits adaptive immune responses should be effective in CIA, adjuvant arthritis or antigen-induced arthritis, as disease in these models is mediated by antigen-specific immune responses by $T$ and $B$ lymphocytes. No other way has yet been found to evaluate the potential of the myriad of therapeutic targets. In vitro studies, computer modeling and experiments in silico have potential, but we need more validation and information on in vivo relevance before launching expensive, time consuming and potentially toxic clinical development programs.

Although assessment of antirheumatic drugs in animal models is still an essential component of drug development, they need to be used judiciously. I generally do not recommend profiling a compound extensively with multiple models. A few simple experiments in systems that test relevant pathways and evaluate the effects on innate and adaptive responses are usually sufficient to make a decision. Evaluation in 'active' and 'passive' models can provide information on the mechanism of action, as can studies that compare efficacy when treatment is initiated before symptom onset versus well-established disease.

Regardless of the approach, it takes a leap of faith to study a compound in humans on the basis of a combination of in vitro studies, in vivo animal experiments and our understanding of the pathogenesis of RA. The risk of toxicity and inadequate efficacy in human trials can be decreased with experiments in animal models, but until 'RA in a mouse' is produced, we can only use these studies to decrease, rather than eliminate, that risk. 